\title{
Serviços de governo eletrônico no Brasil: uma análise sobre fatores de impacto na decisão de uso do cidadão
}

\author{
LUiz Claudio Mendes Vargas ${ }^{1}$ \\ MARIE ANNE MACADAR ${ }^{1}$ \\ Peter Fernandes WANKe ${ }^{1}$ \\ JORge JUNio MOREIRA ANTUNES ${ }^{1}$ \\ ${ }^{1}$ UniVERsidade FEDERAL do RIO de JANEIRO (UFRJ/COPPEAD), RIO DE JANEIRO - RJ, BRASIL
}

\begin{abstract}
Resumo
Este artigo trata dos fatores que influenciam a utilização de serviços de governo eletrônico pelos cidadãos. A literatura acadêmica tem promovido reflexões sobre a influência de diversos fatores, e este artigo tem como objetivo avaliar se aspectos sociodemográficos têm influência sobre a decisão de uso de serviços de governo eletrônico pelos cidadãos brasileiros. O estudo utilizou uma abordagem metodológica quantitativa, valendo-se de testes estatísticos e modelo de regressão logística baseados em microdados da pesquisa TIC Domicílios, coordenada pelo Centro Regional de Estudos para o Desenvolvimento da Sociedade da Informação (Cetic.br), referente ao ano-base de 2019. Os resultados desta análise indicam que há influência significativa de fatores como idade, renda familiar, condição de atividade, classe econômica, grau de instrução, tipo de dispositivo de acesso e uso de serviços de e-commerce sobre a probabilidade de uso de serviços de governo eletrônico. O artigo contribui para o entendimento mais abrangente dos fatores que impactam a demanda e se propõe a servir de insumo para discussões entre gestores públicos sobre a implementação desses serviços.
\end{abstract}

Palavras-chave: Governo eletrônico. TIC Domicílios. Regressão logística.

\section{Electronic Government services in Brazil: an analysis of impact factors on the citizen's usage decision}

\begin{abstract}
This article focuses on factors that influence the use of electronic government services by citizens. The academic literature has promoted reflections on the influence of various factors and, therefore, this article aims to assess whether socio-demographic factors influence the Brazilian citizens' decision to use Electronic Government services. This study adopted a quantitative methodological approach, using statistical tests and a logistic regression model, based on the microdata of the ICT Household 2019 survey, coordinated by the Regional Center of Studies for the Development of the Information Society (CETIC.br). The results indicate a significant influence of age, family income, activity condition, economic class, level of education, type of access device, and use of e-commerce services on the probability of using Electronic Government services by Brazilian citizens. The article contributes to a more comprehensive understanding of the factors that impact demand and aims to subsidize public managers' discussions about these services' implementation.
\end{abstract}

Keywords: Electronic Government. ICT Household. Logistic Regression.

\section{Servicios de gobierno electrónico en Brasil: un análisis de los factores de impacto en la decisión de uso del ciudadano}

\section{Resumen}

Este artículo trata de los factores que influyen en el uso de los servicios de administración electrónica por parte de los ciudadanos. La literatura académica ha promovido reflexiones sobre la influencia de diversos factores y, por lo tanto, este artículo tiene por objeto evaluar si los factores sociodemográficos influyen en la decisión de los ciudadanos brasileños de utilizar los servicios de gobierno electrónico. En esto estudio se usó un enfoque metodológico cuantitativo, valiéndose de pruebas estadísticas y un modelo de regresión logística, basado en los microdatos de la investigación TIC Domicilios, coordinada por el Centro Regional de Estudios para el Desarrollo de la Sociedad de la Información (Cetic.br), para el año base 2019. Los resultados de este análisis indican que hay una influencia significativa de factores como edad, ingresos familiares, condición de la actividad, clase económica, nivel de educación, tipo de dispositivo de acceso y la utilización de servicios de comercio electrónico en la probabilidad de utilizar servicios de gobierno electrónico. El artículo contribuye a una comprensión más completa de los factores que inciden en la demanda y se propone servir de aportación a debates entre los gestores públicos sobre la implantación de estos servicios.

Palabras clave: Gobierno electrónico. TIC Domicilios. Regresión logística. 


\section{INTRODUÇÃO}

Este artigo trata da utilização das tecnologias de informação e comunicação (TIC) por parte de brasileiros que buscam um serviço público governamental, procurando avaliar se aspectos sociodemográficos têm influência sobre a decisão de uso por meio digital (serviços eletrônicos em governo).

Nos últimos anos, a discussão sobre a aplicação das TIC tem ocupado o centro dos debates sobre inovação, tanto no setor privado quanto no público. O termo "transformação digital" se tornou o objetivo de empresas e de governos, tendo a oferta de serviços digitais como principal foco. No setor público, o termo "governo eletrônico (e-gov)" passou a integrar as agendas das autoridades governamentais e a englobar uma vasta gama de serviços e aplicações que têm a tecnologia digital e a internet como plataformas de desenvolvimento e acesso aos usuários.

O conceito de governo eletrônico não está relacionado apenas a aplicar novas tecnologias para prestação de serviços públicos. A ideia é que, por meio da inovação tecnológica - no caso, a aplicação das TIC -, os governos possam prestar serviços que criem valor público e sejam centrados no cidadão. A criação de valor público representa a visão da administração pública para a geração de valor para o cidadão, sendo similar ao conceito de geração de valor agregado para clientes, muito utilizado nas disciplinas de marketing e estratégia (Lopes, Macadar \& Luciano, 2018). Esse é um conceito amplo, que apresenta como principais domínios a melhoria dos processos do setor público, da relação entre governos e cidadãos e da relação entre governos e outras instituições.

A vertente mais difundida acerca do conceito de e-gov está relacionada aos serviços prestados aos cidadãos. No Brasil, por exemplo, os serviços de declaração anual de ajuste do imposto de renda de pessoas físicas, de serviços de agendamento de consultas médicas, de matrículas na rede pública de ensino, de orçamento participativo digital e de centrais de atendimento ao cidadão são casos reconhecidos como experiências bem-sucedidas de ações de governo eletrônico (Araujo, Reinhard \& Cunha, 2018).

A evolução das ações de governo eletrônico apresenta desafios de oferta e da demanda. Pelo lado da oferta, os governos precisam desenvolver uma estratégia para aplicação das TIC que considere as diversas esferas de gestão, investimentos, custos operacionais e a perspectiva de valor para o cidadão. Pelo lado da demanda, é importante entendermos as condições necessárias e as habilidades que o cidadão precisa desenvolver para se tornar ativo usuário de serviços eletrônicos públicos. Nesse contexto, o presente estudo pretende contribuir para o entendimento dos fatores que impactam o lado da demanda por serviços de governo eletrônico.

O estudo se propõe a responder à seguinte pergunta: quais fatores sociodemográficos influenciam a decisão de uso de serviços de governo eletrônico no Brasil? Considerando que a pesquisa sobre o tema tem aplicado de forma predominante o método qualitativo (Cunha, Coelho \& Przeybilovic, 2017), o presente artigo visa contribuir para a literatura acadêmica, com uma abordagem quantitativa, que testa a dependência de serviços de governo eletrônico de condições contextuais e sociodemográficas, baseados num modelo de regressão.

Após esta Introdução, o artigo traz uma 2a seção, dedicada à revisão de literatura sobre os conceitos de serviço eletrônico em governo, a contribuição dos serviços de governo eletrônico para a administração pública e os fatores que influenciam o uso de serviços de governo eletrônico. A 3ạ seção apresenta a abordagem metodológica aplicada, e, em seguida, 2 seções (4⿳亠口了 e 5ạ) são dedicadas à apresentação dos resultados obtidos e à discussão sobre os achados desta análise. Por fim, a 6a seção apresenta as considerações finais. 


\section{REVISÃO DE LITERATURA}

\section{Conceito de Serviço Eletrônico em Governo}

Governo eletrônico é um termo relacionado com o uso das tecnologias de informação e comunicação pelo setor público, inicialmente associado à adoção de sistemas de informação nos processos internos e que depois se expandiu para a aplicação da tecnologia digital para o provimento de serviços aos cidadãos. O termo tem um sentido amplo, uma vez que abrange a aplicação das TIC em diferentes estágios em que a tecnologia impacta o setor público, seja pela digitalização de documentos e processos, seja pela transformação da organização interna, do engajamento dos stakeholders e do direcionamento das políticas públicas (Janowski, 2015).

Não há um consenso na literatura sobre a definição de governo eletrônico, mas os estudos realizados sobre o tema, em geral, abordam a aplicação das TIC para melhoria dos processos e da gestão no setor público, para a melhoria na prestação de serviços ao cidadão e para aumentar o engajamento da sociedade nas discussões de política pública e democracia. Nesse contexto, os programas de governo eletrônico podem abranger 3 diferentes escopos: e-public services, e-public administration e e-democracy (Barbosa, Pozzebon \& Diniz, 2013). Em outras palavras, estamos falando de 3 focos de ação voltados, respectivamente, à prestação de serviços ao público, à administração pública em geral e à promoção da democracia por meio da tecnologia digital ou eletrônica.

Os programas de governo eletrônico não estão restritos à oferta de serviços públicos on-line via internet, entretanto e-public services têm se tornado sua dimensão mais popular e de maior impacto na sociedade. Além disso, essa dimensão é extremamente relevante graças ao potencial de mudar a percepção do cidadão em relação à qualidade dos serviços prestados pelo setor público (Barbosa et al., 2013).

Quando avaliamos as principais causas para a adoção de TIC pelos governos, é possível entender por que a prestação de serviços on-line tem se apresentado como a dimensão mais popular de governo eletrônico. $O$ aumento do uso de tecnologia por cidadãos, empresas privadas e organizações governamentais; a substituição da informação em papel por mídias eletrônicas; e o avanço da própria infraestrutura pública de TIC são apontados como principais motivadores da adoção de TIC de forma intensiva pelo setor público nos processos administrativos internos e na prestação de serviços à sociedade (Przeybilovicz, Cunha \& Coelho, 2015).

O tema governo eletrônico está mais próximo do cidadão e das empresas quando falamos de prestação de serviços via internet, uma vez que se assemelha ao uso de tecnologia para obtenção de serviços privados, como comércio eletrônico, entrega de refeições, contratação de transporte privado, bancários, entre outros serviços que têm se tornado cada vez mais populares. Sob a óptica da caracterização da forma de prestação de serviço, podemos considerar o serviço eletrônico como internet-based, interativo, centrado no cliente e integrado com relação à tecnologia e a processos internos da organização fornecedora. Dessa forma, no contexto de governo eletrônico, os serviços normalmente estão relacionados a bens intangíveis, como troca de informações para receber permissões, benefícios, declarar imposto ou questões similares (Lindgren \& Jansson, 2013).

A popularização de serviços como a entrega eletrônica de declaração de imposto de renda, do voto eletrônico e da concessão de auxílio emergencial para combate à crise causada pela pandemia de COVID-19 levou à associação do termo governo eletrônico, fundamentalmente à dimensão de e-public services (Barbosa et al., 2013). Nesse contexto, os serviços eletrônicos em governo têm sido alvo de estudos e pesquisas que procuram identificar os atributos necessários à sua oferta e os fatores que motivam seu uso por parte dos cidadãos.

Para o objetivo deste estudo, o escopo de governo eletrônico será restrito à dimensão de e-public services, ou seja, de provimento de serviços públicos, por meio eletrônico, a cidadãos e empresas.

\section{Serviços de Governo Eletrônico e sua Contribuição para a Administração Pública}

Governo eletrônico representa mais do que prover serviços digitais para o público. Está também relacionado ao uso de TIC para atender às necessidades dos cidadãos e gerar maior envolvimento da sociedade civil com o governo, em todas as suas esferas. A ampliação do uso de novas tecnologias por pessoas e empresas é uma motivação para aplicação de tecnologias emergentes no setor público, que têm alto potencial inovador. Mas, além disso, devemos considerar os projetos de governo eletrônico sob a óptica do atendimento às necessidades e aos interesses do cidadão. 
Para entender a contribuição proporcionada pelas iniciativas de governo eletrônico, precisamos mencionar os conceitos teóricos da administração pública. Em meados do século XX, o traditional public administration era uma ideia predominante na administração pública. A gestão pública estava voltada a solucionar os desafios de industrialização, urbanização e falhas de mercado. O principal valor era a eficiência operacional dos governos, e os cidadãos eram simplesmente eleitores ou clientes (Bryson, Crosby \& Bloomberg, 2014). A experiência bem-sucedida na recuperação econômica pós-guerras mundiais e grande depressão solidificou a noção de Estado como principal agente do bem-estar geral da sociedade.

A partir da década de 1980, emergiu o conceito de New Public Management (NPM), baseado no entendimento de que a racionalidade econômica dos mercados privados era mais eficaz e que a atuação do Estado como prestador de serviços deveria ser limitada, incentivando a descentralização estatal e a privatização. Era o conceito de governo mais ágil, responsável, transparente, orientado para resultados, descentralizado e eficiente, o que significava um governo que trabalha melhor e com menos custos (Bryson et al., 2014; Cordella \& Bonina, 2012).

As TIC têm papel importante no contexto de NPM, pois se tornam uma ferramenta para criar novas formas de prestação de serviços públicos, mais eficientes e com maior transparência, assim como transforma os procedimentos operacionais e o modelo de gestão pública. Nesse conceito de NPM, a implementação de programas de governo eletrônico estava direcionada pelo racional de custo-benefício, oriundo do modelo de gestão privada. As TIC se tornaram um elemento central no processo de reformulação dos governos, cujo conceito de NPM propunha (Cordella \& Bonina, 2012).

A crítica ao NPM se concentrou no fato de que a gestão pública não deveria se pautar exclusivamente no racional custo-benefício, como no setor privado, uma vez que temas como resposta a desastres naturais, saúde, educação e desigualdade social não poderiam ser tratados com esse mesmo racional. Os resultados produzidos pelas reformas inspiradas no conceito de NPM geraram impactos político-sociais, não abordados pelo racional privado.

Surge, então, uma nova visão de administração pública, baseada nos conceitos de New Public Service (NPS) e public value, nos quais os cidadãos, com suas preferências e necessidades, se tornam o principal objetivo. O conceito de NPS defende que o objetivo do Estado deveria ser servir, em vez de direcionar (server, rather than steer), ou seja, o papel principal do setor público seria apoiar os cidadãos na busca pelo atendimento aos seus interesses comuns, e não controlar e direcionar os rumos da sociedade por meio de uma lógica de economia de mercado (Denhardt \& Denhardt, 2000). O NPS dialoga com o conceito de public value, que também ganhou relevância como contraponto aos objetivos do NPM. Nesse contexto de public value, o objetivo da administração pública deveria ser criar valor para o cidadão, mediante prestação de serviços públicos, regulação, promoção da democracia e da igualdade, entre outras ações (Lopes et al., 2018; Moore, 1994).

Com base nos conceitos de NPS e public value, o governo eletrônico passou a ser considerado um meio para criação de valor para os cidadãos, atendendo as suas preferências coletivas. Enquanto entre as décadas de 1980 e 2000, no contexto do NPM, as iniciativas de governo eletrônico foram impulsionadas por experiências do setor privado, nas últimas décadas houve uma mudança do debate, para como entregar valor público por serviços de governo eletrônico.

Mais recentemente, as discussões sobre desenvolvimento sustentável e combate à pobreza e às desigualdades passaram a ter maior influência sobre a evolução do tema governo eletrônico. Os compromissos estabelecidos entre os países membros da Organização das Nações Unidas (ONU), a Agenda 2030 para o Desenvolvimento Sustentável e os Objetivos de Desenvolvimento Sustentável (ODS) têm influência sobre os direcionamentos da política de aplicação de TIC pelos governos. A tecnologia digital é considerada um fator-chave para a implementação dos ODS (Janowski, 2016). Essas ferramentas são tão importantes quanto os ODS propriamente ditos, de tal forma que um dos 17 objetivos tem foco na articulação dessas ferramentas e caminhos de implementação (Marcovecchio, Thinyane, Estevez \& Janowski, 2019). Há um grande foco na discussão dos caminhos para implantação, o que passa por governança pública e tecnologia, entre outros temas.

Dessa forma, para garantir que as iniciativas de governo eletrônico sejam eficazes como ferramentas para o atingimento dos ODS, é fundamental entender como a situação sociodemográfica dos cidadãos impacta o uso dos serviços de governo eletrônico. Para que os objetivos sejam atingidos de forma plena e abrangente, é importante que atinjam toda a população, a despeito de condição socioeconômica. Mas, para que os serviços de governo eletrônico sejam um instrumento para a promoção desses objetivos, é necessário não haver restrições de uso em função de gênero, classe social, nível de escolaridade, região de habitação e outros fatores. 


\section{Fatores que Influenciam o Uso de Serviços de Governo Eletrônico}

A adoção de inovações tecnológicas no setor público e a adesão por parte dos cidadãos aos programas de governo eletrônico têm sido objeto de estudos acadêmicos no Brasil e no exterior. Em geral, esses estudos têm como foco a análise dos atributos de aceitação da tecnologia por potenciais usuários, usando como referencial teórico os modelos Technological Acceptance Model (TAM) (Davis, 1989) e Unified Theory of Acceptance and Use of Technology (UTAUT) (Venkatesh, Morris, Gordon \& Davis, 2003).

Alguns estudos apontam fatores como facilidade de uso, segurança, infraestrutura necessária e disponibilidade de suporte técnico como principais determinantes do uso de serviços de governo eletrônico (Shareef, V. Kumar, U. Kumar \& Dwivedi, 2014; Van Dijk, Peters \& Ebbers, 2008; Venkatesh, Chan \& Thong, 2012). Outros estudos, ao usarem a abordagem das capacidades (Capabilities Approach), desenvolvida por Amartya Sen (1999), apresentam elementos de relacionamento entre as TIC e o desenvolvimento humano. $O$ desenvolvimento deve ser percebido como a expansão das capacidades dos seres humanos que devem levar a vida de acordo com seus valores -, nas quais as TIC devem ser consideradas meios para alcançar o objetivo de desenvolvimento humano (Zheng, 2009).

Nessa linha, é possível trabalhar sob uma abordagem que vai além dos atributos inerentes ao serviço e que de alguma forma apresenta fatores influenciadores do uso de serviços de governo eletrônico. Esse é o caso de estudos brasileiros que consideraram, como variáveis explicativas de seus modelos, fatores como a classe social e o local preferido para acesso à internet (Araujo et al., 2018; Araujo \& Reinhard, 2015; Santos \& Reihardt, 2015).

Os estudos elaborados por Araujo e Reinhard (2015) e por Araujo et al. (2018) apresentam abordagens similares. Neles, os autores utilizam os dados da pesquisa para compreender como o uso de serviços de governo eletrônico é influenciado pelo local de acesso à internet e pelas competências dos usuários. Já o artigo elaborado por Santos e Reinhard (2015) traz uma abordagem um pouco diferente, com uma análise exploratória sobre o uso e as motivações do não uso de serviços de governo eletrônico no Brasil. Por meio de uma análise baseada em estatísticas descritivas das respostas apresentadas na TIC Domicílios 2019 (Comitê Gestor da Internet no Brasil [CGI], 2020), os autores identificam os principais fatores apontados pelos entrevistados como fatores de não uso.

A inclusão de características sociodemográficas na avaliação de fatores que influenciam no uso de serviços de governo eletrônico foi internacionalmente trabalhada por Van Dijk et al. (2008). Inspirado no modelo UTAUT, os autores propõem um framework multidisciplinar em que a intenção de uso é determinada pelos atributos de serviço apresentados no modelo original e por fatores sociodemográficos como idade, gênero, grau de escolaridade e ocupação. Entre os estudos realizados no cenário internacional, Barrera-Barrera, Rey-Moreno \& Medina-Molina (2019) é o que considera o perfil sociodemográfico na análise de uso dos serviços de governo eletrônico.

Valendo-se de dados da pesquisa realizada pelo Centro de Investigações Sociológicas (CIS) da Espanha e aplicando a técnica de análise multivariada conhecida como tabela de contingência (contingency tables), Barrera-Barrera et al. (2019) concluíram que o perfil sociodemográfico tem influência sobre a preferência de uso de serviços de governo eletrônico. No entanto, o potencial impacto de características e condições sociodemográficas dos usuários, que podem influenciar nos serviços de governo eletrônico, ainda não foi academicamente analisado no contexto brasileiro.

Adicionalmente, podem-se acrescentar atributos como confiança na internet e no governo (Belanger \& Carter, 2008); facilidade de uso, segurança, suporte técnico (Venkatesh et al., 2012); crença religiosa, percepção de utilidade, resistência às mudanças (Alomari, 2014); consciência, qualidade da informação, disponibilidade de recursos (Shareef et al., 2014); influência social, hábito, benefícios percebidos (Moraes \& Meirelles, 2017). O Quadro 1 apresenta uma relação de estudos realizados e os atributos considerados com potenciais influenciadores do uso de serviços de governo eletrônico. 
Quadro 1

Atributos influenciadores do uso de serviços de governo eletrônico

\begin{tabular}{|c|c|}
\hline Autores & Atributos \\
\hline $\begin{array}{l}\text { Van Dijk et al. } \\
\qquad(2008)\end{array}$ & $\begin{array}{l}\text { Influência social, atitude em relação ao uso, performance } \\
\text { esperada, esforço esperado, opção de canal de preferência, } \\
\text { acesso à mídia digital, experiência com mídia digital, oferta } \\
\text { de serviços, idade, gênero, grau de escolaridade e ocupação. }\end{array}$ \\
\hline $\begin{array}{l}\text { Bélanger e Carter } \\
\text { (2008) }\end{array}$ & Confiança na internet e no governo. \\
\hline $\begin{array}{l}\text { Venkatesh et al. } \\
\qquad(2012)\end{array}$ & $\begin{array}{l}\text { Facilidade de uso, recursos de informática, suporte técnico } \\
\text { e segurança. }\end{array}$ \\
\hline $\begin{array}{l}\text { Alomari } \\
(2014)\end{array}$ & $\begin{array}{l}\text { Confiança na internet, website design, crença religiosa, } \\
\text { habilidades de informática, boca a boca, resistência às } \\
\text { mudanças, percepção de utilidade, complexidade e utilização. }\end{array}$ \\
\hline $\begin{array}{l}\text { Shareef et al. } \\
\qquad(2014)\end{array}$ & $\begin{array}{l}\text { Consciência, disponibilidade de recursos, habilidades para } \\
\text { uso multi-idioma, qualidade da informação, confiança, } \\
\text { segurança, benefícios percebidos e imagem. }\end{array}$ \\
\hline $\begin{array}{l}\text { Araujo e Reinhard } \\
\text { (2015) }\end{array}$ & $\begin{array}{l}\text { Classe social, local de acesso à internet, uso de e-commerce, } \\
\text { de e-financial services e de e-mail. }\end{array}$ \\
\hline $\begin{array}{l}\text { Santos e Reihard } \\
\qquad(2015)\end{array}$ & $\begin{array}{l}\text { Preferência por contato pessoal, dificuldade de acesso, } \\
\text { segurança, indisponibilidade de serviços e ausência de } \\
\text { retorno das solicitações. }\end{array}$ \\
\hline $\begin{array}{l}\text { Moraes e Meirelles } \\
\qquad(2017)\end{array}$ & $\begin{array}{l}\text { Benefícios percebidos, condições facilitadoras, facilidade } \\
\text { de uso, influência social, segurança, confiança e hábito. }\end{array}$ \\
\hline $\begin{array}{l}\text { Araujo et al. } \\
\qquad(2018)\end{array}$ & $\begin{array}{l}\text { Classe social, local de acesso à internet e uso da rede para } \\
\text { estudo, trabalho, relacionamento e pesquisas. }\end{array}$ \\
\hline $\begin{array}{l}\text { Barrera-Barrera et al. } \\
\qquad(2019)\end{array}$ & $\begin{array}{l}\text { Gênero, idade, grau de instrução, estado civil, condição } \\
\text { de atividade e religião. }\end{array}$ \\
\hline
\end{tabular}

Fonte: Elaborada pelos autores.

Em resumo, observa-se que somente 3 estudos incorporaram variáveis sociodemográficas nas suas análises - Araujo e Reinhard (2015), Araujo et al. (2018), Van Dijk et al. (2008) -, permitindo ampliar a discussão sobre os fatores que influenciam o uso de serviços de governo eletrônico e trazendo à tona as condições e as capacidades do cidadão para se tornar usuário desses serviços. O contexto do Brasil foi abordado em dois artigos (Araujo et al., 2018; Araujo \& Reinhard, 2015), mas características sociodemográficas como idade, renda, grau de instrução, gênero e região de residência não foram consideradas. Dessa forma, entendemos que a realização de uma análise considerando a inclusão de tais variáveis proporcionará uma contribuição relevante e complementar aos estudos já realizados no Brasil, permitindo compreender melhor o perfil sociodemográfico do usuário de serviços de governo eletrônico e traçar políticas públicas que contemplem esse perfil de cidadão.

\section{METODOLOGIA}

Para analisar que fatores têm influência no uso de serviços de governo eletrônico no Brasil, o presente estudo segue uma abordagem metodológica quantitativa, baseada na pesquisa TIC Domicílios 2019, elaborada pelo Cetic.br, consistindo, portanto, numa análise de dados secundários. Considerando que o objetivo deste artigo é avaliar que fatores influenciam a decisão de uso de serviços de governo eletrônico, temos uma análise de decisão que pode ser traduzida nos resultados de uma variável dependente binária ou dicotômica com 2 opções: 1, se o indivíduo utiliza serviços de governo eletrônico, ou 0, se não utiliza.

Em outras palavras, o estudo visa avaliar a probabilidade de um indivíduo ser usuário de serviços de governo eletrônico dadas as suas características sociodemográficas, como sexo, idade, renda, classe social e grau de instrução. Dessa forma, o modelo 
de regressão logística é o mais indicado para a avaliação de dependência ou associação de uma variável categórica binária em relação a outras variáveis qualitativas ou quantitativas, uma vez que consiste numa forma especializada de regressão que visa prever e explicar o comportamento de uma variável que assume 2 posições específicas apenas, não sendo uma medida dependente (Hair, Black, Babin, Anderson \& Tatham, 2009).

De modo geral, o modelo considera que a variável dependente $y$ pode assumir 2 valores:

$$
y_{i}=\left\{\begin{array}{l}
1, \text { se há ocorrência de determinado evento } \\
\text { ou } \\
0, \text { se não há ocorrência }
\end{array}\right.
$$

A probabilidade de ocorrência do evento em análise é estimada no modelo com base na equação:

$$
P\left(y_{i}=1\right)=\frac{1}{1+e^{-g(x)}}
$$

Em que $g(x)=+\alpha+\beta_{0} \cdot x_{i}$.

\section{COLETA DE DADOS}

A pesquisa TIC Domicílios tem como objetivo medir o acesso às TIC nos domicílios brasileiros e seu uso pela população. Trata-se de uma pesquisa com abrangência nacional, cujo público-alvo são domicílios particulares permanentes e indivíduos com 10 anos ou mais. As respostas foram coletadas presencialmente, no período entre outubro de 2019 e março 2020. A coleta dos dados foi realizada com um formulário eletrônico aplicado por meio de tablet, método denominado computer-assisted personal interviewing (Capi).

Além das variáveis contextuais e sociodemográficas, a pesquisa TIC Domicílios 2019 coletou dados para indicadores específicos, agrupados em 9 módulos temáticos:

- Módulo A: Acesso às tecnologias de informação e comunicação no domicílio.

- Módulo B: Uso de computadores.

- Módulo C: Uso da internet.

- Módulo G: Governo eletrônico.

- Módulo H: Comércio eletrônico.

- Módulo I: Habilidades com o computador.

- Módulo J: Uso de telefone celular.

- Módulo L: Uso de aplicações selecionadas.

- Módulo TC: Atividades culturais.

O Módulo G, que aborda a temática de governo eletrônico, apresenta 29 questões relacionadas ao perfil de uso de serviços de governo eletrônico. As respostas a essas questões foram agregadas numa única variável, denominada G1_Agreg, que indica se o usuário usou ou não serviços de governo eletrônico.

Essa decisão se reflete no resultado da variável G1_Agreg, que indica se o entrevistado utilizou (G1_Agreg = 1: Sim) ou não (G1_Agreg = 0: Não). A hipótese deste estudo é que outras variáveis podem influenciar tal decisão. 


\section{PROCEDIMENTO PARA TRATAMENTO E ANÁLISE DOS DADOS}

O método definido para análise das variáveis que podem influenciar a decisão de uso de serviços de governo eletrônico contempla as etapas descritas no Quadro 2, a seguir.

Quadro 2

Etapas do processo de tratamento e análise

\begin{tabular}{|l|l|}
\hline \multicolumn{1}{|c|}{ Etapas } & \multicolumn{1}{c|}{ Objetivos } \\
\hline $\begin{array}{l}\text { Análise e escolha preliminar das variáveis consideradas } \\
\text { na base da pesquisa TIC Domicílios 2019. }\end{array}$ & $\begin{array}{l}\text { Identificar as variáveis da base da pesquisa TIC Domicílios } \\
2019 \text { que têm potencial para influenciar a decisão de uso } \\
\text { de serviços de governo eletrônico. }\end{array}$ \\
\hline Tratamento da base de dados. & $\begin{array}{l}\text { Adequar a base de dados para a elaboração de testes, análises } \\
\text { estatísticas e modelo de regressão. }\end{array}$ \\
\hline $\begin{array}{l}\text { Testes e análises estatísticas para conhecimento do grau } \\
\text { de associação entre as variáveis explicativas e a variável } \\
\text { dependente. }\end{array}$ & $\begin{array}{l}\text { Com base nas variáveis previamente identificadas, definir as } \\
\text { que serão usadas para a análise de regressão, em função do } \\
\text { grau de associação com a variável dependente. }\end{array}$ \\
\hline Análise de regressão, utilizando o modelo Logit. & $\begin{array}{l}\text { Utilizando a regressão logística, estimar a equação que } \\
\text { determina a probabilidade de uso de serviços de governo } \\
\text { eletrônico face à ocorrência das demais condições. }\end{array}$ \\
\hline
\end{tabular}

Fonte: Elaborado pelos autores.

Essas etapas foram realizadas utilizando-se o software estatístico livre R ( $R$ Core Team, 2017). A seguir, o estudo apresenta um detalhamento de cada uma das etapas.

\section{ANÁLISE E ESCOLHA PRELIMINAR DAS VARIÁVEIS}

A base de dados da TIC Domicílios 2019 (indivíduos) contém 20.536 respostas de questionários com 249 questões, o que representa 20.536 observações e 249 variáveis. Para os objetivos deste estudo, as variáveis relevantes são aquelas relacionadas com a caraterização socioeconômica do indivíduo, presentes nos seguintes grupos do dicionário de variáveis da pesquisa TIC Domicílios 2019: informações do domicílio, uso da internet, comércio eletrônico, governo eletrônico, cobertura e variáveis agregadas - esta apresenta informações agregadas de variáveis constantes nos demais.

Considerando o grupo informações do domicílio, foram escolhidas as variáveis "sexo", "idade" e "renda familiar". As variáveis "PEA" (condição de atividade) e "grau de instrução" foram substituídas por "PEA 2" e "grau de instrução 2", do grupo cobertura, que são versões com rótulos ajustados das anteriores.

No grupo uso da internet, foi escolhida a variável C1, que corresponde a questão "o respondente já usou a internet?" Como será apresentado posteriormente, essa variável serve de filtro, uma vez que o uso de serviços de governo eletrônico abordado neste estudo depende do acesso à internet.

A variável $\mathrm{H} 2$ foi escolhida entre as apresentadas no grupo comércio eletrônico e indica se o respondente comprou ou encomendou produtos ou serviços pela internet nos últimos 12 meses. No grupo telefone celular, foi escolhida a variável J5, que indica se o respondente tem celular.

As variáveis do grupo governo eletrônico foram substituídas pela variável agregada G1_Agreg, presente no grupo de variáveis agregadas, conforme mencionado antes. No grupo cobertura, além das variáveis PEA_2 e Grau_Instrucao_2, foram escolhidas as variáveis COD_Regiao_2, Classe_CB2015 e Area, que indicam, respectivamente, região geográfica, classe econômica e área urbana/rural do indivíduo.

Por fim, no grupo variáveis agregadas, além de G1_Agreg, também foi escolhida a variável C5_Dispositivos, que informa se o indivíduo utilizou computador, celular ou ambos para acesso à internet. O Quadro 3, abaixo, apresenta um sumário com as variáveis escolhidas, de forma preliminar, para compor o modelo de análise deste estudo. 
Quadro 3

Variáveis escolhidas para o modelo de análise

\begin{tabular}{|c|c|c|c|c|c|}
\hline ID_variável & Descrição da variável & Tipo de dado & Código e rótulo da variável & Classificação & Categoria \\
\hline Sexo & Sexo & Numérico & $\begin{array}{l}1=\text { Masculino } \\
2=\text { Feminino }\end{array}$ & Quali & Nominal \\
\hline Idade & $\begin{array}{l}\text { Idade do } \\
\text { respondente }\end{array}$ & Numérico & & Quanti & Contínua \\
\hline PEA_2 & $\begin{array}{l}\text { Condição de atividade: faz parte } \\
\text { da População Economicamente } \\
\text { Ativa (PEA) }\end{array}$ & Numérico & $\begin{array}{l}1=\text { PEA } \\
2=\text { Não PEA }\end{array}$ & Quali & Nominal \\
\hline $\mathrm{H} 2$ & $\begin{array}{l}\text { Compra ou encomenda de } \\
\text { produtos ou serviços pela } \\
\text { internet }\end{array}$ & Numérico & $\begin{array}{l}0=\text { Não } \\
1=\text { Sim } \\
97 \text { = Não sabe } \\
98 \text { = Não respondeu } \\
99 \text { = Não se aplica }\end{array}$ & Quali & Nominal \\
\hline J5 & Tem celular & Numérico & $\begin{array}{l}0=\text { Não } \\
1=\operatorname{Sim} \\
97=\text { Não sabe } \\
98=\text { Não respondeu }\end{array}$ & Quali & Nominal \\
\hline $\begin{array}{l}\text { Renda_- } \\
\text { Familiar }\end{array}$ & Renda familiar & Numérico & $\begin{array}{l}1=\text { Até } R \$ 937,00 \\
2=\text { De } R \$ 937,01 \text { até } R \$ 1.874,00 \\
3=\text { De } R \$ 1.874,01 \text { até } R \$ 2.811,00 \\
4=\text { De } R \$ 2.811,01 \text { até } R \$ 4.685,00 \\
5=\text { De } R \$ 4.685,01 \text { até } R \$ 9.370,00 \\
6=\text { De } R \$ 9.370,01 \text { até } R \$ 18.740,00 \\
7=\text { De } R \$ 18.740,01 \text { até } R \$ 28.110,00 \\
8=\text { Mais de } R \$ 28.110,00 \\
9=\text { Não tem renda } \\
97=\text { Não sabe } \\
98=\text { Não respondeu }\end{array}$ & Quali & Ordinal \\
\hline $\begin{array}{l}\text { Classe_- } \\
\text { CB2015 }\end{array}$ & $\begin{array}{l}\text { Classe econômica pelo Critério } \\
\text { Brasil } 2015\end{array}$ & Numérico & $\begin{array}{l}1=A \\
2=B \\
3=C \\
4=D E\end{array}$ & Quali & Ordinal \\
\hline $\begin{array}{c}\text { Grau_ } \\
\text { Instrucao_2 }\end{array}$ & $\begin{array}{l}\text { Grau de instrução informado } \\
\text { pelo respondente }\end{array}$ & Numérico & $\begin{array}{l}\text { 1= Analfabeto/Educação infantil } \\
2=\text { Fundamental } \\
3 \text { = Médio } \\
4 \text { = Superior }\end{array}$ & Quali & Ordinal \\
\hline$\frac{\mathrm{C5}_{-}}{\text {Dispositivos }}$ & $\begin{array}{l}\text { Dispositivo utilizado pelos } \\
\text { usuários de internet de forma } \\
\text { exclusiva ou simultânea }\end{array}$ & Numérico & $\begin{array}{l}1=\text { Apenas computador } \\
2=\text { Apenas telefone celular } \\
3=\text { Ambos } \\
99=\text { Não se aplica }\end{array}$ & Quali & Nominal \\
\hline Area & Área urbana ou rural & Numérico & $\begin{array}{l}1=\text { Urbana } \\
2=\text { Rural }\end{array}$ & Quali & Nominal \\
\hline $\begin{array}{c}\text { COD__ } \\
\text { Regiao_2 }\end{array}$ & Região Geográfica & Numérico & $\begin{array}{l}1=\text { Sudeste } \\
2=\text { Nordeste } \\
3=\text { Sul } \\
4=\text { Norte } \\
5=\text { Centro-Oeste }\end{array}$ & Quali & Nominal \\
\hline
\end{tabular}

Fonte: Elaborado pelos autores. 


\section{TRATAMENTO DA BASE DE DADOS}

O tratamento da base foi realizado com apoio do software estatístico livre R ( $R$ Core Team, 2017), conforme representado na Figura 1, a seguir.

A base de microdados da pesquisa TIC Domicílios 2019 foi extraída do site do Cetic.br, no formato de planilha em Excel. Em seguida, a base de microdados foi carregada no software estatístico livre R utilizando o pacote readxl (Wickham \& Bryan, 2019). Foi necessário realizar alguns filtros e criar um dataframe apenas com as variáveis predefinidas para a análise. Para esse procedimento, instalaram-se os pacotes gmodels (Warnes, Bolker, Lumley \& Johnson, 2018) e dplyr (Wickham, François, Henry \& Müller, 2020). Após execução das etapas do processo de tratamento, a base de dados ficou reduzida a 11.781 observações e 12 variáveis.

Figura 1

Processo de extração e tratamento dos dados

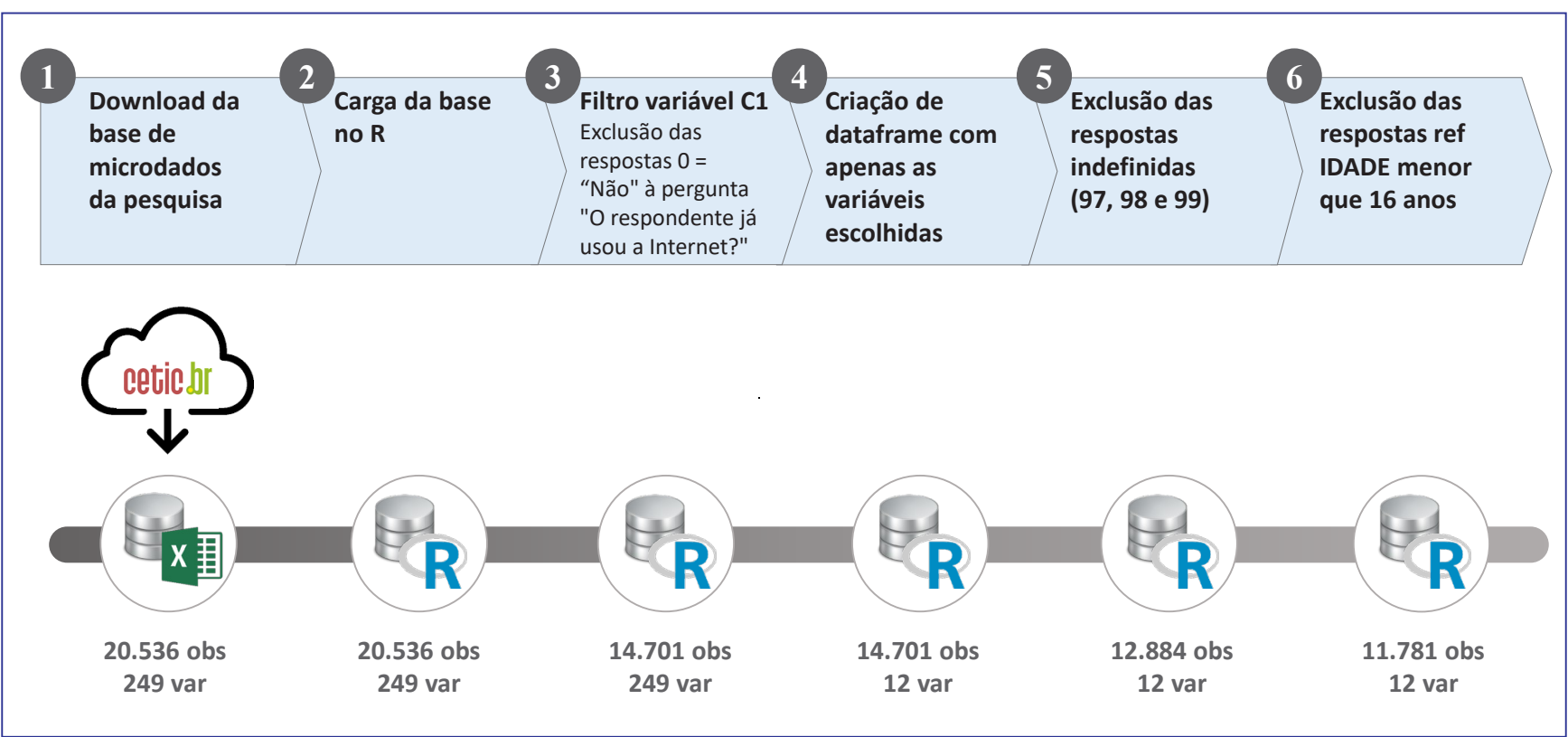

Fonte: Elaborada pelos autores.

Para efeito dos testes de associação, foi necessário excluir as respostas indefinidas (codificadas como 97, 98 e 99) para as variáveis Renda_Familiar, C5_Dispositivos, $\mathrm{H} 2$ e J5. Os questionários cujos respondentes informaram idade inferior a 16 anos foram excluídos, tendo em vista que os serviços de governo eletrônico, em geral, se destinam a pessoas com 16 anos ou mais.

Além desses filtros, foi necessário adaptar as codificações de algumas variáveis para a elaboração dos testes de associação e do modelo de regressão, conforme apresentado no Quadro 4. 
Quadro 4

Adaptações nas codificações das variáveis

\begin{tabular}{|c|c|c|c|}
\hline \multirow{2}{*}{ ID_variável } & \multirow{2}{*}{$\begin{array}{l}\text { Descrição } \\
\text { da variável }\end{array}$} & \multicolumn{2}{|c|}{ Código e rótulo da variável } \\
\hline & & $\mathrm{De}$ & Para \\
\hline PEA_2 & $\begin{array}{l}\text { Condição de atividade: } \\
\text { faz parte da População } \\
\text { Economicamente Ativa (PEA) }\end{array}$ & $\begin{array}{l}1=\text { PEA } \\
2=\text { Não PEA }\end{array}$ & $\begin{array}{l}0=\text { Não PEA } \\
1=\text { PEA }\end{array}$ \\
\hline $\begin{array}{l}\text { Renda_- } \\
\text { Familiar }\end{array}$ & Renda familiar & $\begin{array}{l}1=\text { Até } R \$ 937,00 \\
2=\text { De } R \$ 937,01 \text { até } R \$ 1.874,00 \\
3=\text { De } R \$ 1.874,01 \text { até } R \$ 2.811,00 \\
4=\text { De } R \$ 2.811,01 \text { até } R \$ 4.685,00 \\
5=\text { De } R \$ 4.685,01 \text { até } R \$ 9.370,00 \\
6=\text { De } R \$ 9.370,01 \text { até } R \$ 18.740,00 \\
7=\text { De } R \$ 18.740,01 \text { até } R \$ 28.110,00 \\
8=\text { Mais de } R \$ 28.110,00 \\
9=\text { Não tem renda }\end{array}$ & $\begin{array}{l}0=\text { Não tem renda } \\
1=\text { Até } R \$ 937,00 \\
2=\text { De } R \$ 937,01 \text { até } R \$ 1.874,00 \\
3=\text { De } R \$ 1.874,01 \text { até } R \$ 2.811,00 \\
4=\text { De } R \$ 2.811,01 \text { até } R \$ 4.685,00 \\
5=\text { De } R \$ 4.685,01 \text { até } R \$ 9.370,00 \\
6=\text { De } R \$ 9.370,01 \text { até } R \$ 18.740,00 \\
7=\text { De } R \$ 18.740,01 \text { até } R \$ 28.110,00 \\
8=\text { Mais de } R \$ 28.110,00\end{array}$ \\
\hline Classe_CB2015 & $\begin{array}{l}\text { Classe econômica pelo } \\
\text { Critério Brasil } 2015\end{array}$ & $\begin{array}{l}1=A \\
2=B \\
3=C \\
4=D E\end{array}$ & $\begin{array}{l}0=D E \\
1=C \\
2=B \\
3=A\end{array}$ \\
\hline Area & Área urbana ou rural & $\begin{array}{l}1=\text { Urbana } \\
2=\text { Rural }\end{array}$ & $\begin{array}{l}0=\text { Rural } \\
1=\text { Urbana }\end{array}$ \\
\hline
\end{tabular}

Fonte: Elaborado pelos autores.

\section{TESTES E ANÁLISES ESTATÍSTICAS}

Após a escolha das variáveis com potencial de impacto sobre o uso de serviços de governo eletrônico e tratamento da base de dados, foi elaborada uma análise de independência entre a variável dependente G1_Agreg e as demais variáveis explicativas. Tendo em vista as variáveis explicativas qualitativas e quantitativas, 3 tipos de ferramentas foram aplicados, conforme a combinação das variáveis dependente (qualitativa) e explicativa (quantitativa ou qualitativa).

\section{G1_Agreg versus Variável Qualitativa}

Nesse caso, foram analisadas as tabelas de associação entre as variáveis e os testes qui-quadrado e V de Cramer. Foram definidas as seguintes hipóteses para os testes de independência:

- HO: as variáveis são independentes.

- H1: as variáveis não são independentes.

Considerando que a distribuição qui-quadrado não é simétrica, com intervalo de confiança (IC) de 95\%, ou seja, nível de significância igual a 5\%, a hipótese nula (H0) será aceita quando $p$-valor $>0,05$.

As análises foram elaboradas no R, utilizando a função CrossTable() para a elaboração das tabelas de associação e a função assocstats(), do pacote vcd (Meyer, Zeileis \& Hornik, 2020), para o cálculo das estatísticas dos testes qui-quadrado e $V$ de Cramer.

\section{G1_Agreg versus Variável Quantitativa}

Nesse caso, foram analisadas medidas de estatística descritiva e os gráficos de distribuição e boxplot para identificação de provável associação entre as variáveis, seguindo as técnicas de análise exploratória de dados (Bussab \& Morettin, 2010). As estatísticas e os gráficos foram gerados no $R$. 


\section{Matriz de Correlação}

De forma complementar às ferramentas de análise indicadas acima, foi elaborada uma matriz de correlação entre as variáveis, baseada no método de Spearman, mais indicado para a avaliação envolvendo variáveis qualitativas. A matriz foi gerada no R, utilizando a função pairs.panel(), no pacote psych (Revelle, 2020).

\section{ANÁLISE DE REGRESSÃo}

Com base nos resultados de testes e análises estatísticas, definiram-se as variáveis que apresentam probabilidade significativa de associação com a variável dependente uso de governo eletrônico (G1_Agreg). Em seguida, o estudo aplica o modelo de regressão logística para estimar a decisão de uso de serviços de governo eletrônico com base em variáveis contextuais e sociodemográficas.

Para o cálculo da regressão logística, foi necessário transformar as variáveis Renda_Familiar, Classe_CB2015, Grau_Instrucao_2 e C5_Dispositivos em variáveis dummy. Para isso, foi criado um novo dataframe no R, utilizando a função dummy_cols(), do pacote fastDummies (Kaplan, 2020). O cálculo da regressão logística foi realizado sobre o dataframe que continha as variáveis dummy, utilizando a função multinom(), do pacote nnet (Venables \& Ripley, 2002). A estimativa das respostas do modelo para a variável dependente foi realizada com auxílio da função predict(), do pacote caret (Kuhn, 2020).

Para finalizar, a performance do modelo foi avaliada com base na elaboração de uma matriz de confusão (confusion matrix), que consiste numa tabela associativa de respostas estimadas versus respostas reais e na análise dos indicadores de acurácia (Accuracy e F-score) e da curva ROC (Kubat, 2017; Kuhn \& Johnson, 2013; Tharwat, 2018). Para elaborar a matriz de confusão, foi utilizada a função confusionMatrix(), do pacote caret (Kuhn, 2020), que também fornece os resultados dos indicadores Accuracy e F-score. Para a análise da curva ROC, foram utilizadas as funções roc() e auc(), do pacote $p$ ROC (Robin et al., 2011).

\section{RESULTADOS}

Os resultados de testes e análise da correlação entre a variável dependente e as demais variáveis qualitativas estão apresentados no Quadro 5, a seguir.

Quadro 5

Resultados dos testes das variáveis qualitativas

\begin{tabular}{|c|c|c|c|c|c|}
\hline ID_variável & Descrição & $\begin{array}{l}\text { Teste Qui } \\
p \text {-valor }\end{array}$ & Teste V & Correlação & Conclusão \\
\hline Sexo & Sexo & $\begin{array}{c}p \text {-value }=1.154 \mathrm{e}-11 \\
\text { Rejeita HO }\end{array}$ & 0,063 & $-0,06$ & $\begin{array}{l}\text { Não é } \\
\text { relevante }\end{array}$ \\
\hline PEA_2 & $\begin{array}{c}\text { Condição de atividade: faz parte da } \\
\text { População Economicamente Ativa (PEA) }\end{array}$ & $\begin{array}{c}\text { p-value }=4.251 \mathrm{e}-94 \\
\text { Rejeita HO }\end{array}$ & 0,190 & $+0,19$ & Relevante \\
\hline $\mathrm{H} 2$ & $\begin{array}{c}\text { Compra ou encomenda de produtos ou } \\
\text { serviços pela internet }\end{array}$ & $\begin{array}{c}p \text {-value }=6.764 \mathrm{e}-309 \\
\text { Rejeita } \mathrm{HO}\end{array}$ & 0,346 & +035 & Relevante \\
\hline J5 & Posse de telefone celular & $\begin{array}{c}p \text {-value }=1.297 \mathrm{e}-14 \\
\text { Rejeita HO }\end{array}$ & 0,071 & $+0,07$ & $\begin{array}{l}\text { Não é } \\
\text { relevante }\end{array}$ \\
\hline $\begin{array}{l}\text { Renda_ } \\
\text { Familiar }\end{array}$ & Renda familiar & $\begin{array}{c}p \text {-value }=4.66 \mathrm{e}-121 \\
\text { Rejeita } \mathrm{HO}\end{array}$ & 0,223 & $+0,22$ & Relevante \\
\hline Classe_CB2015 & Classe econômica pelo Critério Brasil 2015 & $\begin{array}{c}p \text {-value }=2.285 \mathrm{e}-138 \\
\text { Rejeita } \mathrm{HO}\end{array}$ & 0,233 & $+0,23$ & Relevante \\
\hline$\underset{\text { Instrucao_2 }}{\text { Grau_ }}$ & $\begin{array}{l}\text { Grau de instrução informado pelo } \\
\text { respondente }\end{array}$ & $\begin{array}{c}p \text {-value }=9.762 \mathrm{e}-276 \\
\text { Rejeita } \mathrm{HO}\end{array}$ & 0,329 & $+0,32$ & Relevante \\
\hline
\end{tabular}




\begin{tabular}{|c|c|c|c|c|c|}
\hline ID_variável & Descrição & $\begin{array}{l}\text { Teste Qui } \\
p \text {-valor }\end{array}$ & Teste V & Correlação & Conclusão \\
\hline$\frac{\text { C5_ }}{\text { Dispositivos }}$ & $\begin{array}{l}\text { Dispositivo utilizado pelos usuários de } \\
\text { internet de forma exclusiva ou simultânea }\end{array}$ & $\begin{array}{c}p \text {-value }=4.212 \mathrm{e}-232 \\
\text { Rejeita HO }\end{array}$ & 0,301 & $+0,30$ & Relevante \\
\hline Area & Área urbana ou rural & $\begin{array}{c}p \text {-value }=1.255 \mathrm{e}-19 \\
\text { Rejeita HO }\end{array}$ & 0,084 & $+0,08$ & $\begin{array}{l}\text { Não é } \\
\text { relevante }\end{array}$ \\
\hline COD_Regiao_2 & Região geográfica & $\begin{array}{c}p \text {-value }=2.658 \mathrm{e}-09 \\
\text { Rejeita HO }\end{array}$ & 0,062 & $-0,03$ & $\begin{array}{l}\text { Não é } \\
\text { relevante }\end{array}$ \\
\hline
\end{tabular}

Fonte: Elaborado pelos autores.

O resultado do teste qui-quadrado indicou a rejeição da hipótese nula $(\mathrm{HO})$, ou seja, de independência entre a variável que representa uso de governo eletrônico e as demais variáveis qualitativas. Entretanto, os resultados do teste $V$ de Cramer e da correlação apresentaram valores abaixo de 0,1 para as variáveis que representam sexo, posse de celular, área urbana ou rural e região geográfica, o que indica uma relação de associação fraca entre as variáveis.

Com base nesses resultados, essas variáveis foram descartadas para a análise de regressão. Havia uma dúvida quanto à variável que representa área urbana ou rural, pois, apesar de os resultados dos testes sugerirem que a associação entre esta e a variável uso de governo eletrônico seja fraca, a tabela de associação entre tal variável e a variável dependente G1_Agreg apresenta uma inversão na proporção entre usuários e não usuários de governo eletrônico quando há mudança de área rural para urbana, conforme apresentado na Tabela 1. Entretanto, apesar disso, decidiu-se por excluir essa variável da análise de regressão.

Tabela 1

Tabela de associação: uso de serviços de e-gov e área rural/urbana

\begin{tabular}{l|c|c|c}
\cline { 2 - 4 } \multicolumn{1}{c|}{} & \multicolumn{3}{|c}{ Área rural / urbana } \\
\hline Uso de serviços e-gov & $\begin{array}{c}\text { Area }=0 \\
\text { (rural) }\end{array}$ & $\begin{array}{c}\text { Area }=1 \\
\text { (urbana) }\end{array}$ & $\begin{array}{c}\text { Total } \\
\text { linha }\end{array}$ \\
\hline G1_Agreg = 0 & 462 & 3.700 & 4.162 \\
(Não) & $48,8 \%$ & $34,1 \%$ & 7.619 \\
\hline G1_Agreg =1 & 484 & 7.135 & $\mathbf{1 1 . 7 8 1}$ \\
(Sim) & $51,2 \%$ & $65,9 \%$ & $\mathbf{1 0 . 8 3 5}$ \\
\hline Total coluna & $\mathbf{9 4 6}$ & & \\
\hline
\end{tabular}

Fonte: Elaborada pelos autores.

Para a variável quantitativa Idade, foram calculadas as estatísticas descritivas, apresentadas na Tabela 2, e elaborada a análise de correlação, apresentado no Quadro 6. Os resultados dos testes indicam que há relação de dependência entre a variável que representa uso de governo eletrônico e a variável Idade.

Tabela 2

Estatísticas da variável quantitativa

\begin{tabular}{c|c|c|c}
\hline Uso de Serviços de e-gov & Média & Desvio-padrão & Mediana \\
\hline G1_Agreg = 0 (Não) & 43,38 & 16,54 & 43 \\
\hline G1_Agreg $=1(\mathrm{Sim})$ & 36,13 & 14,40 & 33 \\
\hline
\end{tabular}

Fonte: Elaborada pelos autores. 


\section{Resultados dos testes da variável quantitativa}

\begin{tabular}{|c|c|c|c|c|}
\hline ID_variável & Descrição & $\begin{array}{c}\text { Estatística descritiva } \\
\text { Análise gráfica }\end{array}$ & Correlação & Conclusão \\
\hline Idade & $\begin{array}{c}\text { Idade do } \\
\text { respondente }\end{array}$ & $\begin{array}{c}\text { A idade dos usuários é menor } \\
\text { que a dos não usuários }\end{array}$ & $-0,15$ & Relevante \\
\hline
\end{tabular}

Fonte: Elaborado pelos autores.

A análise de regressão apresentou os seguintes resultados.

\begin{tabular}{|c|c|c|c|c|c|}
\hline & Estimate & Std. Error & $z$ value & $\operatorname{Pr}(>|z|)$ & \\
\hline (Intercept) & -1.0532948 & 0.3161304 & -3.3318 & 0.0008627 & $* * *$ \\
\hline Idade & -0.0215132 & 0.0015091 & -14.2557 & $<2.2 \mathrm{e}-16$ & $* * *$ \\
\hline PEA_2 & 0.5584534 & 0.0524883 & 10.6396 & $<2.2 \mathrm{e}-16$ & $* * *$ \\
\hline Classe_CB2015_1 & 0.2105292 & 0.0525329 & 4.0076 & $6,135 \mathrm{E}-02$ & $* * *$ \\
\hline Classe_CB2015_2 & 0.2379410 & 0.0936015 & 2.5421 & 0.0110200 & * \\
\hline Classe_CB2015_3 & 0.3481345 & 0.2775756 & 1.2542 & 0.2097704 & \\
\hline Grau_Instrucao_2_1 & 0.4283330 & 0.1228478 & 3.4867 & 0.0004890 & $* * *$ \\
\hline Grau_Instrucao_2_2 & 0.9302344 & 0.1242343 & 7.4877 & $7,007 \mathrm{E}-11$ & $* * *$ \\
\hline Grau_Instrucao_2_3 & 1.1445511 & 0.1410431 & 8.1149 & $4,862 \mathrm{E}-13$ & $* * *$ \\
\hline C5_Dispositivos_2 & 0.4207865 & 0.2358820 & 1.7839 & 0.0744423 & . \\
\hline C5_Dispositivos_3 & 1.0915803 & 0.2395162 & 4.5574 & $5,178 \mathrm{E}-03$ & $* * *$ \\
\hline $\mathrm{H} 2$ & 1.0552418 & 0.0542522 & 19.4507 & $<2.2 \mathrm{e}-16$ & $* * *$ \\
\hline Renda_Familiar_1 & -0.0060700 & 0.1600424 & -0.0379 & 0.9697457 & \\
\hline Renda_Familiar_2 & 0.1893167 & 0.1604950 & 1.1796 & 0.2381673 & \\
\hline Renda_Familiar_3 & 0.4414333 & 0.1653371 & 2.6699 & 0.0075874 & $* *$ \\
\hline Renda_Familiar_4 & 0.3680310 & 0.1712689 & 2.1488 & 0.0316464 & * \\
\hline Renda_Familiar_5 & 0.4954595 & 0.1895137 & 2.6144 & 0.0089392 & $* *$ \\
\hline Renda_Familiar_6 & 0.4227457 & 0.2604689 & 1.6230 & 0.1045856 & \\
\hline Renda_Familiar_7 & 0.3334971 & 0.4863970 & 0.6856 & 0.4929351 & \\
\hline Renda_Familiar_8 & 0.0349981 & 0.5717860 & 0.0612 & 0.9511932 & \\
\hline
\end{tabular}


O modelo considera que a variável dependente G1_Agreg (uso de serviços de governo eletrônico) pode assumir dois valores:

$$
y_{i}\left(\mathrm{G} 1 \_ \text {Agreg }\right)=\left\{\begin{array}{l}
1, \text { se o indivíduo utiliza serviços de governo eletrônico } \\
\text { ou } \\
0, \text { se não utiliza }
\end{array}\right.
$$

A probabilidade de ocorrência do evento "uso de serviços de governo eletrônico", portanto, foi estimada no modelo com base na equação:

$$
P\left(y_{i}=1\right)=\frac{1}{1+e^{-g(x)}}
$$

Em que: $\mathrm{g}(\mathrm{x})=-1.0532948-0.0215132 \times($ Idade $)+0.5584534 \mathrm{x}$ (PEA_2)

$$
\begin{aligned}
& +0.2105292 \times \text { (Classe_CB2015_1)+0.2379410 x (Classe_CB2015_2) } \\
& +0.3481345 \times \text { (Classe_CB2015_3)+0.4283330 } \times \text { (Grau_Instrucao_2_1) } \\
& +0.9302344 \times \text { (Grau_Instrucao_2_1)+1.1445511 x (Grau_Instrucao_2_3) } \\
& +0.4207865 \times \text { (C5_Dispositivos_2)+1.0915803 x (C5_Dispositivos_3) } \\
& +1.0552418 \times \text { (H2)-0.0060700 } \times \text { (Renda_Familiar_1) } \\
& +0.1893167 \times \text { (Renda_Familiar_2)+0.4414333 } \times \text { (Renda_Familiar_3) } \\
& +0.3680310 \times \text { (Renda_Familiar_4)+0.4954595 } \times \text { (Renda_Familiar_5) } \\
& +0.4227457 \times \text { (Renda_Familiar_6)+0.3334971 x (Renda_Familiar_7) } \\
& +0.0349981 \times \text { (Renda_Familiar_8) }
\end{aligned}
$$

Conforme descrito antes, na seção de metodologia, a performance do modelo de regressão logística aplicado foi avaliada pela elaboração da matriz de confusão. Os resultados estão apresentados na Tabela 3.

A análise indicou que o modelo apresenta uma resposta ajustada, com nível aceitável de acurácia, conforme pode ser observado pelo resultado dos indicadores Accuracy e F-score, apresentados na Tabela 4.

Tabela 3

Resultados da matriz de confusão

\begin{tabular}{c|c|c|c}
\cline { 2 - 4 } & \multicolumn{3}{|c}{ Observações reais } \\
\hline Valores previstos pelo modelo & $\begin{array}{c}\text { G1_Agreg }=0 \\
\text { (Não) }\end{array}$ & $\begin{array}{c}\text { G1_Agreg }=1 \\
\text { (Sim) }\end{array}$ & Total linha \\
\hline $\begin{array}{c}\text { Resultado estimado }=0 \\
\text { (Não) }\end{array}$ & 2.196 & 1.198 & 3.394 \\
\hline $\begin{array}{c}\text { Resultado estimado = } \\
\text { (Sim) }\end{array}$ & 1.966 & 6.421 & 8.387 \\
\hline Total coluna & $\mathbf{4 . 1 6 2}$ & $\mathbf{7 . 6 1 9}$ & $\mathbf{1 1 . 7 8 1}$ \\
\hline
\end{tabular}

Fonte: Elaborada pelos autores. 
Tabela 4

Resultados dos indicadores de performance do modelo

\begin{tabular}{c|c}
\hline Indicadores & Resultado \\
\hline Accuracy & 0,7314 \\
\hline F-score (F1) & 0,8023 \\
\hline Recall & 0,8428 \\
\hline Precision & 0,7656 \\
\hline
\end{tabular}

Fonte: Elaborada pelos autores.

Em resumo, o modelo de regressão logística apresentou uma resposta satisfatória em relação à estimativa de decisão de um indivíduo usar ou não os serviços de governo eletrônico, confirmando a hipótese de associação entre a variável dependente e as variáveis explicativas escolhidas.

\section{DISCUSSÃO}

Os resultados da análise indicam que há uma influência significativa de idade, renda familiar, condição de atividade, classe econômica, grau de instrução, tipo de dispositivo de acesso e uso de serviços de e-commerce sobre a probabilidade de uso de serviços de governo eletrônico. Por outro lado, não há influência significativa em função de gênero, tipo de área (urbana ou rural) e região geográfica.

Os testes e as análises estatísticas preliminares serviram para confirmar quais variáveis deveriam ser consideradas no modelo de regressão logística. Dessa forma, a baixa correlação entre as variáveis que representavam gênero, tipo de área (urbana ou rural) e região geográfica levou à conclusão de que elas deveriam ser excluídas. Os serviços de governo eletrônico servem para qualquer cidadão, independentemente de gênero.

O perfil dos respondentes que indicaram ter utilizado serviços de governo eletrônico e dos que indicaram não ter utilizado não apresenta diferenças significativas em relação à sua região geográfica. Já em relação ao tipo de área do domicílio, urbana ou rural, apesar de o percentual de usuários em área rural ser inferior ao de usuários em área urbana, a quantidade de observações referentes aos respondentes da pesquisa em área urbana é muito superior à quantidade de observações referentes aos respondentes em área rural. Esse viés implica uma correlação baixa entre essa variável e a variável dependente. Em teoria, a disponibilidade de infraestrutura de acesso à internet em áreas rurais é menor do que nas urbanas, o que levaria ao entendimento de que esse seria um fator relevante para a análise. Entretanto, a concentração elevada das observações em área urbana não permitiu que tal condição fosse verificada.

De acordo com o estudo, os usuários mais jovens são mais propensos ao uso de serviços de governo eletrônico. Isso pode estar relacionado com uma maior habilidade dos jovens em lidar com recursos tecnológicos.

Fazer parte da PEA tem impacto positivo sobre a probabilidade de uso de serviços de governo eletrônico. Esse resultado pode estar relacionado com o fato, por exemplo, de trabalhadores ativos utilizarem serviços prestados pela Receita Federal, como declaração de imposto de renda, ao contrário daqueles que não têm ocupação.

Quanto à renda familiar, indivíduos das faixas de renda mais altas apresentam maior probabilidade de uso de serviços de governo eletrônico. O mesmo se observa em relação à classe econômica e ao grau de instrução. Em tese, renda, classe econômica e grau de instrução estão associadas, indicando que uma maior renda está associada a um grau de instrução mais elevado, implicando em classe econômica mais elevada. Indivíduos de classes econômicas mais altas têm mais acesso a computador, celular e internet. Além disso, tendem a ter mais habilidade para usar serviços digitais.

A correlação entre ser usuário de serviços e-commerce e de governo eletrônico pode ser explicada pela similaridade entre as condições para o acesso aos dois tipos de serviço, como dispositivos e capacidades para o uso. 
Por fim, o modelo aponta que adotar o celular como dispositivo de acesso, unicamente ou em conjunto com o computador, tem impacto muito significativo na probabilidade de utilização de serviços de governo eletrônico. A hipótese é que há maior divulgação e facilidade de uso de serviços digitais via aplicativos para celulares.

Dessa forma, podemos entender que a demanda por serviços de governo eletrônico aumentará à medida que houver investimentos em educação, emprego e renda da população, buscando equiparar as condições econômicas de áreas rurais às das áreas urbanas e melhorar a qualidade de acesso à internet via celular.

\section{CONSIDERAÇÕES FINAIS}

O presente estudo procurou avaliar se fatores sociodemográficos têm influência sobre a decisão de uso de serviços de governo eletrônico pelos cidadãos brasileiros. Para atingir esse objetivo, adotou-se uma abordagem quantitativa, tendo como base a pesquisa TIC Domicílios 2019, realizada pelo Cetic.br. A metodologia aplicada para a realização da análise teve como base o modelo de regressão logística, tendo em vista o comportamento da variável dependente que assume apenas 2 posições específicas, isto é, uma variável binária (Hair et al., 2009).

Os resultados obtidos pela análise dos dados e do modelo de regressão logística aplicado demonstram que as condições sociodemográficas têm influência sobre a decisão de uso dos serviços de governo eletrônico. Conforme a hipótese inicial, questões como nível de escolaridade, nível de renda, idade etc. têm influência significativa na escolha do cidadão por utilizar ou não os serviços de governo eletrônico.

O presente estudo cumpre seu objetivo de contribuir para a literatura acadêmica com uma abordagem quantitativa, que testa a dependência do uso de serviços de governo eletrônico em relação às condições contextuais e sociodemográficas, baseado num modelo de regressão logística. Além disso, reforça a necessidade de considerarmos as variáveis contextuais e sociodemográficas nos modelos de análise de adoção de tecnologia pelos governos.

Nesse contexto, os gestores públicos responsáveis pela estratégia de aplicação das TIC e, consequentemente, pelo desenvolvimento de programas de governo eletrônico devem levar em conta essas características sociodemográficas para a criação de políticas públicas. De igual modo, é importante considerar as diferenças sociodemográficas nas avaliações de resultados dos programas implementados. O sucesso na aceitação de determinado serviço de governo eletrônico não depende só das características técnicas intrínsecas do serviço, mas também das características sociodemográficas que definem o perfil do público-alvo, ou o perfil padrão do cidadão que será usuário desse serviço.

Recomenda-se que estudos futuros considerem uma nova análise, considerando um número maior de observações da área rural, no sentido de testar a hipótese de que a variável sociodemográfica referente ao tipo de área de domicílio tem influência sobre o uso de serviços de governo eletrônico. Adicionalmente, considerando que a variável dependente escolhida representa uma consolidação de 29 questões da pesquisa TIC Domicílios relacionadas ao perfil de uso de serviços de governo eletrônico, recomenda-se, como proposta para estudos futuros, uma análise mais específica, com base em alguns tipos de serviço ou características de uso específicas. Pode-se também comparar os resultados desta análise com outras similares realizadas em distintos países.

Como recomendação final, porém não exaustiva, este estudo pode ser ampliado, considerando a análise dos microdados da pesquisa TIC Domicílios baseada na utilização de referencial teórico do campo das teorias de sistemas de informação, construindo, assim, um modelo conceitual de análise que incorpore os fatores sociodemográficos como influenciadores do uso de serviços de governo eletrônico. 


\section{REFERÊNCIAS}

Alomari, M. K. (2014). Discovering Citizens Reaction Toward E-Government: Factors in E-Government Adoption. Journal of Information Systems and Technology Management, 11(1), 5-20.

Araujo, M. H., \& Reinhard, N. (2015). Factors influencing the use of electronic government services in Brazil. Lecture Notes in Informatics (LNI). Proceedings - Series of the Gesellschaft fur Informatik (GI), 221(4), 140-149.

Araujo, M. H., Reinhard, N., \& Cunha, M. A. (2018). Serviços de governo eletrônico no Brasil: uma análise a partir das medidas de acesso e competências de uso da internet. Revista de Administração Pública, 52(4), 676-694.

Barbosa, A. F., Pozzebon, M., \& Diniz, E. H. (2013). Rethinking E-government performance assessment from a citizen perspective. Public Administration, 91(3), 744-762.

Barrera-Barrera, R., Rey-Moreno, M., \& Medina-Molina, C. (2019). Explanatory factors of the preference and use of electronic administration in Spain. Journal of Public Administration, 53 (2), 349-374.

Bélanger, F., \& Carter, L. (2008). Trust and risk in e-government adoption. Journal of Strategic Information Systems, 17(2), 165-176.

Bryson, J. M., Crosby, B. C., \& Bloomberg, L. (2014). Public Value Governance: Moving beyond Traditional Public Administration and the New Public Management. Public Administration Review, 74(4), 445-456.

Bussab, W. O., \& Morettin, P. A. (2010). Estatística Básica (6a ed.). São Paulo, SP: Saraiva.

Comitê Gestor da Internet no Brasil. (2020). Pesquisa sobre o uso das tecnologias da informação e comunicação no Brasil: pesquisa TIC Domicílios, ano 2019: Relatório de coleta de dados. São Paulo, SP: Autor.

Cordella, A., \& Bonina, C.M. (2012). A public value perspective for ICT enabled public sector reforms: A theoretical reflection. Government Information Quarterly, 29, 512-520.

Cunha, M. A., Coelho, T. R., \& Przeybilovicz, E. (2017). Get into the club: positioning a developing country in the international e-gov research. The Electronic Journal of Information Systems in Developing Countries, 79, 1-21.

Davis, F. D. (1989). Perceived usefulness, perceived ease of use, and user acceptance of information technology. MIS Quarterly: Management Information Systems, 13(3), 319-339.

Denhardt, R. B., \& Denhardt, J.V. (2000). The New Public Service: Server rather than Steering Public Administration Review, 60(6), 549-559.

Hair, J. F., Jr., Black, W. C., Babin, B. J., Anderson, R. E., \& Tatham, R. L. (2009). Análise Multivariada de Dados (6a Ed.). Porto Alegre, RS: Bookman

Janowski, T. (2015). Digital government evolution: From transformation to contextualization. Government Information Quarterly, 32(3), 221-236.
Janowski, T. (2016). Implementing Sustainable Development Goals with Digital Government - Aspiration-capacity gap. Government Information Quarterly, 33, 603-613.

Kaplan, J. (2020). fastDummies: Fast Creation of Dummy (Binary). Columns and Rows from Categorical Variables. $R$ package version 1.6.2. Recuperado de https://cran.r-project.org/package=fastDummies

Kubat, M. (2017). An Introduction to Machine Learning. New York, NY: Springer.

Kuhn, M. (2020). caret: Classification and Regression Training. $R$ package version 6.0-86. Recuperado de https://cran.r-project. $\mathrm{org} /$ package=caret

Kuhn, M., \& Johnson, K. (2013). Applied predictive modeling. New York, NY: Springer.

Lindgren, I., \& Jansson, G. (2013). Electronic services in the public sector: A conceptual framework. Government Information Quarterly, 30(2), 163-172.

Lopes, K., Macadar, M. A., \& Luciano, E. (2018). Valor Público: o Cidadão no Centro da Gestão Pública. Pesquisa Sobre o Uso das Tecnologias de Informação e Comunicação no Setor Público Brasileiro 2017. São Paulo, SP: Comitê Gestor da Internet no Brasil.

Marcovecchio, I., Thinyane, M., Estevez, E., \& Janowski, T. (2019). Digital Government as Implementation Means for Sustainable Development Goals. International Journal of Public Administration in the Digital Age, 6(3), 1-22.

Meyer, D., Zeileis, A., \& Hornik, K. (2020). vcd: Visualizing Categorical Data. $R$ package version 1.4-8. Recuperado de https://rdrr.io/cran/vcd/

Moore, M. H. (1994). Public Value as the Focus of Strategy. Australian Journal of Public-Administration, 53(3), 296-303.

Moraes, G. H. D. S. M., \& Meirelles, F. S. (2017). User's perspective of eletronic government adoption in Brazil. Journal of Technology Management and Innovation, 12(2), 1-10.

Przeybilovicz, E., Cunha, M. A., \& Coelho, T. R. (2015).O desenvolvimento dos estudos sobre Governo Eletrônico no Brasil: um estudo bibliométrico e sociométrico. Revista Eletrônica de Sistemas de Informação, 14(3), 3-24.

R Core Team. (2017). R: A language and environment for \#\# statistical computing.R Foundation for Statistical Computing, \#\# Vienna, Austria. Recuperado de https://www.r-project.org/

Revelle, W. (2020). psych: Procedures for Personality and Psychological Research. Northwestern University, Evanston, Illinois, USA. Recuperado de https://cran.r-project.org/package=psych Version $=2.0 .8, \% 0 \mathrm{~A}$

Robin, X ., Turck, N., Hainard, A., Tiberti, N., Lisacek, F., Sanchez, J.C., ... Muller, M.. (2011). pROC: an open-source package for $\mathrm{R}$ and $\mathrm{S}+$ to analyze and compare ROC curves. BMC Bioinformatics, 12(77), 1-8.

Santos, E. M., \& Reinhard, N. (2015). Serviços de Governo Eletrônico: um estudo sobre o uso por indivíduos no Brasil. In Proceedings of 25응 International Conference on Information Resources Management, Ottawa, Ontario. 
Sen, A. (1999). Development as Freedom. Oxford, UK: Oxford University Press.

Shareef, M. A., Kumar, V., Kumar, U., \& Dwivedi, Y. (2014). Factors affecting citizen adoption of transactional electronic government. Journal of Enterprise Information Management, 27(4), 385-401.

Tharwat, A. (2018). Classification assessment methods. Applied Computing and Informatics, 17(1), 168-192.

Van Dijk, J. A. G. M., Peters, O., \& Ebbers, W. (2008). Explaining the acceptance and use of government Internet services: A multivariate analysis of 2006 survey data in the Netherlands. Government Information Quarterly, 25(3), 379-399.

Venables, W. N., \& Ripley, B. D. (2002). Modern Applied Statistics with S. (4a Ed.). New York, NY: Springer.

Venkatesh, V., Chan, F. K. Y., \& Thong, J. Y. L. (2012). Designing e-government services: Key service attributes and citizens' preference structures. Journal of Operations Management, 30(1-2), 116-133.
Venkatesh, V., Morris, M. G., Gordon, B., \& Davis, F. D. (2003). User acceptance of Information Technology: toward a unified view. MIS Quarterly, 27(3), 425-478, 2003.

Warnes, G. R., Bolker, B., Lumley, T., \& Johnson, R. C. (2018). gmodels: Various $R$ Programming Tools for Model Fitting. $R$ package version 2.18.1. Recuperado de https://cran.r-project.org/ package $=$ gmodels

Wickham, H., \& Bryan, J. (2019). readxl: Read Excel Files. $R$ package version 1.3.1. Recuperado de https://cran.r-project.org/ package=readxl

Wickham, H., François, R., Henry, L., \& Müller, K. (2020). dplyr: A Grammar of Data Manipulation. R package version 1.0.2. Recuperado de https://cran.r-project.org/package=dplyr

Zheng, Y. (2009). Different spaces for e-development: What can we learn from the capability approach? Information Technology for Development, 15(2), 66-82.

Luiz Claudio Mendes Vargas

ORCID: https://orcid.org/0000-0002-9410-7153

Doutorando em Administração do COPPEAD da Universidade Federal do Rio de Janeiro (UFRJ). E-mail: luiz.vargas@coppead.ufrj.br

Marie Anne Macadar

ORCID: https://orcid.org/0000-0003-2744-5352

Doutora em Administração e professora adjunta do COPPEAD da Universidade Federal do Rio de Janeiro (UFRJ).E-mail: marie.macadar@coppead.ufrj.br

Peter Fernandes Wanke

ORCID: https://orcid.org/0000-0003-1395-8907

Doutor em Engenharia de Produção e professor titular do COPPEAD da Universidade Federal do Rio de Janeiro (UFRJ). E-mail: peter@coppead.ufrj.br

Jorge Junio Moreira Antunes

ORCID: https://orcid.org/0000-0003-3199-5912

Engenheiro Mecânico e pesquisador do COPPEAD da Universidade Federal do Rio de Janeiro (UFRJ). E-mail: jorge.moreira@coppead.ufrj.br 